Environment Conservation Journal 15(3) 121-126, 2014

ISSN 0972-3099 (Print) 2278-5124 (Online)

Abstracted and Indexed

\title{
Isolation, identification and biological characterization of Acanthamoeba polyphaga from suspected cases of eye patients and its electron microscopy
}

\author{
Newton Paul ${ }^{1} \bowtie$, Tabrez Ahmad ${ }^{2}$ and A.K.Sharma ${ }^{2}$
}

Received:12.07.2014

Accepted:15.11.2014

\begin{abstract}
Corneal scrapings and tear drop of 90 patients with suspected cases of keratitis and eye infection attending the Govt. Hospital and rural areas patients of Lucknow city were screened for amoebic pathogens including Acanthamoeba. Of these, 6 (6.66percent) were positive for Acanthamoeba, which fulfilled the criteria for suspecting Acanthamoeba keratitis. These were distributed among all ages with the maximum numbers in the 20-45 year age group. None of the patients were using contact lenses and were mostly agricultural labourers and had recent history of swimming in ponds and river and also associated with water sports. The predisposing factor found in this study was trauma of varying degrees. The result of present study confirmed that Amphizoic amoebae colonized almost every conceivable aquatic habitat. This is alarming signal that shows presence of Amphizoic amoebae. Incidences of infection during warm season have been traced in patients suffering from eye infection. Preventive measures include public awareness and maintenance of water body and adequate chlorination.
\end{abstract}

\section{Keywords: Acanthamoeba, Keratitis, Amphizoic Amoebae, Chlorination}

\section{Introduction}

Acanthamoeba is a free-living, opportunistic protozoan parasite of human beings. Acanthamoeba can cause a fatal Meningoencephalitis disease, but its few species have been found to be commonly associated with eye infections, typically, Acanthamoeba keratitis associated with contact lens use (Anisah et al, 2005). The increasing prevalence of this eye disease is thought to be linked to the increased use of contact lenses (Kamel et al, 2003). Acanthamoeba keratitis is usually diagnosed after viral and bacterial causes have been eliminated (and, as a result, there is often a significant delay before appropriate treatment is administered). Because of the severity of Acanthamoeba keratitis, a significant loss of visual acuity is common and in many cases total loss of sight in the infected eye occurs (Khan, 2003). Current methods of detection involve the culture and microscopic identification .These methods are time consuming, laborious, and open to error. The development of a rapid, simple detection method for Acanthamoeba is thus important. Acanthamoeba species have been

\section{Author's Address}

${ }^{1}$ Department of Zoology, Isabella Thoburn College, Lucknow .

${ }^{2}$ Protozoology Research laboratory, Department of Zoology,

University of Lucknow, Lucknow U.P. India,

E-mail:newton.mymail@gmail.com isolated from diverse sources, such as freshwater, seawater, chlorinated water from swimming pools, dental treatment units, and contact lens cases (Anisah et al, 2004 and Visvesvara, 2010). Most of the strains found are not pathogenic. Some pathogenic forms are known to survive for extended periods in fresh water. The presence of pathogenic Acanthamoeba organisms in the atmosphere is an important factor in the prevalence of Acanthamoeba keratitis, although this is not its main cause (Siddiqui and Khan, 2012).Patients with Acanthamoeba keratitis usually are users of dailywear disposable, soft contact lenses. The use of tap water to rinse contact lenses allows deposits of lime scale to accumulate, and this lime scale often contains pathogenic Acanthamoeba species. Lenses create a corneal abrasion, facilitating entry of Acanthamoeba. The organism can survive in contact lens cases and solutions.

\section{Material and Methods}

For the diagnosis of Acanthamoeba keratitis, sample (tear or corneal scraping) isolated from the patients of different age group ranging from 3year to 17 years. All samples, tear and corneal scrap were collected in sterile screw capped small tubes 
and brought to the lab and tested for culture on non nutrients agar plate pre seeded with E.coli in accordance with a standard method (Visvesvara,2010). Microscopy and culture for amebic, bacterial, mycobacterial, and fungal organisms were performed. Swabs from the cornea were inoculated onto two Escherichia coli-seeded, $1 \%$ non nutrient agar (ECNNA) plates. In addition; the patient's disposable contact lenses wereplaced onto two ECNNA plates. Ten milliliters of the patient'scontact lens-disinfecting solution was centrifuged at $3,000 \mathrm{rpm}$ for $10 \mathrm{~min}$, and the sedimentwas inoculated onto two ECNNA plates. All of the ECNNA plateswere incubated at 25 and $37^{\circ} \mathrm{C}$ for 20 days. The disinfecting solutionwas cultured forbacteria. The Acanthamoeba isolate was cloned by diluting a suspension of cysts in sterile ameba saline, spreading them on agar undera microscope, and selecting individual cysts by using low magnification A piece of agar bearing the selected cyst was cut outand transferred face down to a fresh ECNNA plate. Several plateswere prepared in this manner from sequential cultures, and eachtime the block of agar was carefully examined under the microscopeto make sure that only one cyst was present.

\section{Results and Discussion}

Acanthamoeba Keratitis is a seldom recorded infection; however, it is a serious condition hence it should not be overlooked or taken lightly. This infection is influential on the eyes and if not treated timely, then it may lead to ocular impairment or blindness on permanent basis (Hammersmith, 2006). It is studied that the infection is caused due to free- living microscopical amoeba which are also known as Acanthamoeba. When these microorganisms, Acanthamoeba, infects the cornea of the eye (external transparent layer of the eye) it leads to Acanthamoeba keratitis. Humans are at greater risk of developing this infection, as these kinds of amoebae are frequently found in various water bodies such as lakes, soil air etc. This condition for first diagnose in the 1973, wherein approximately $90 \%$ of affected individuals were contact lenses users. There are several factors that may lead toAcanthamoeba keratitis. For example, using contaminated water from sources such as tap or well may increase the risk of such infection.
Also infected contact lenses may cause such issues (Shoff et al, 2007). Avoid wearing contact lenses while in hot tub or swimming in pool or even while taking shower as these factors may also result in Acanthamoeba infection (Shoff et al, 2008). Adopting inappropriate measures for storing contact lenses may cause the virus to settle on the lenses and then infect your eyes on wearing them.

Six positive cases out of 90 examined $(6.666 \%)$ for suspected cases of amoebic keratitis showed positive growth from their eye scrapings /tear drops. Identification and biological characterization of amoebic isolates was done following the patterns of Singh and Hanumaiah (1979) and Levine et al (1980). All the trophic characters observed were similar to the typical of Acanthamoeba sp. strain.

However the cyst was double-walled and polyhedral. The endocyst (inner) was stellate or polygonal and ectocyst was wrinkled with ripples. In Scanning Electron Microscopy study, ridges and groves were present on the surface of cyst and pores were present on the cyst wall (Plate-1 fig1, $2 \& 3,4)$. Cysts were uninucleate with a prominent nucleolus. During excystment trophozoite emerged from the pore that was present on cyst wall. Trophozoites also failed to produce temporary amoebo flagellate stage on repeated efforts (Plate2, Fig 1, 2\&3). Thus on the basis of above observations of trophozoites and cysts, the strains isolated from eye of suspected patients were also identified as Acanthamoeba polyphaga because of their close similarity in the observation of trophic, cystic characters and locomotion \& behaviour. Free-living amoebae (FLA) are the main predators of bacterial populations in the environment, playing a major role in maintaining the ecological balance of many environmental systems by feeding on bacterial population, which is present nearly in all water bodies, due to which humans are directly exposed to the risk of amoebic infection through water (Carter, 1968; Culbertson et al, 1972 and Singh, 1985). As already mentioned, these amoebae are ubiquitous in nature and present in all water bodies. During study, samples were taken from the suspected patients of amoebic infection (i.e. cerebrospinal fluid and nasal swab of suspected cases of meningitis and respiratory tract infection and tear drops/eye scrapings from suspected cases of keratitis) Pathogenic free-living amoebae are known as Amphizoic amoebae because they are 

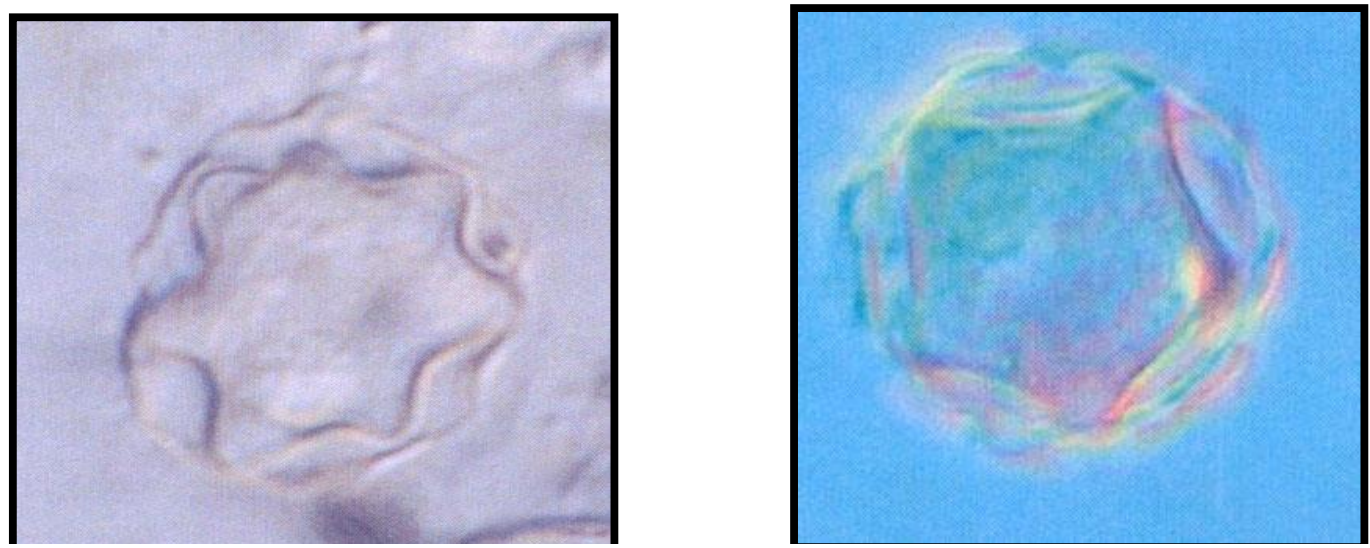

Figure 1 Shows Photographs of Cyst of Acanthamoebae polyphaga
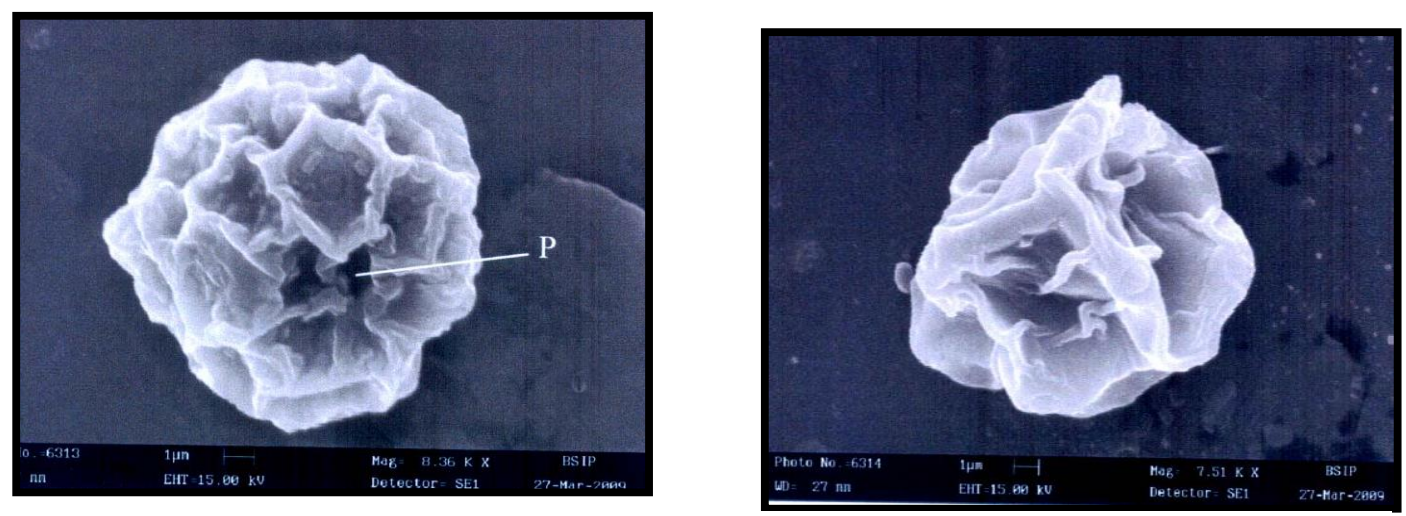

Figure 2 Shows SEM of Acanthamoebapolyphaga
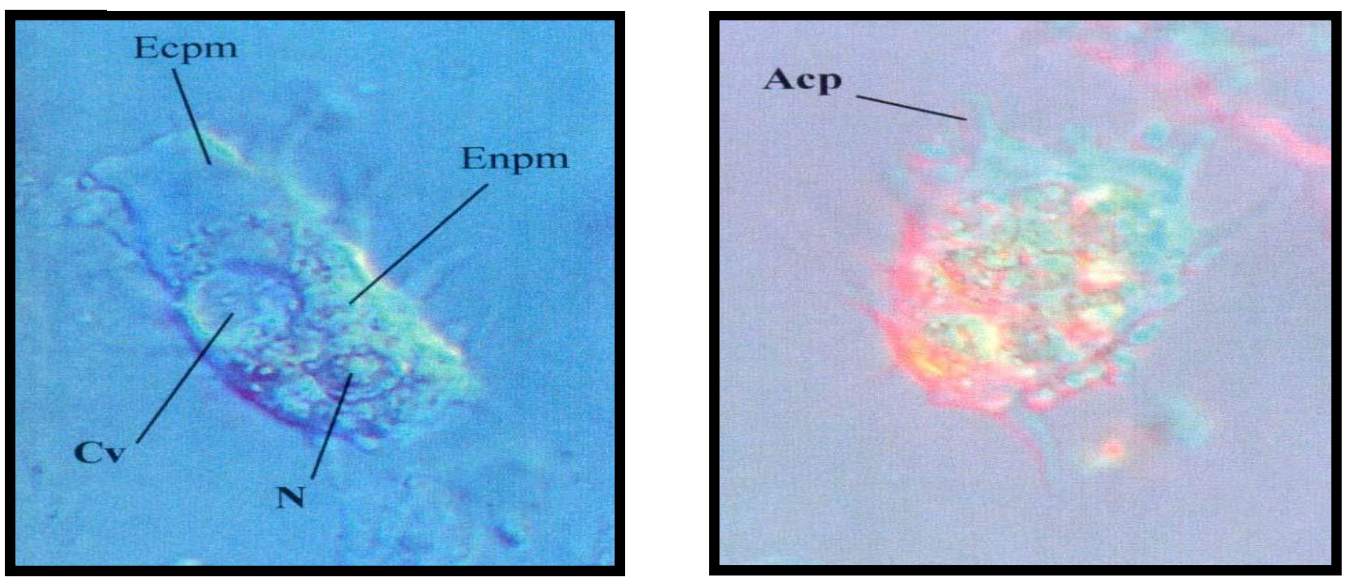

Figure 3 Shows Trophozoite of Acanthamoeba polyphaga 


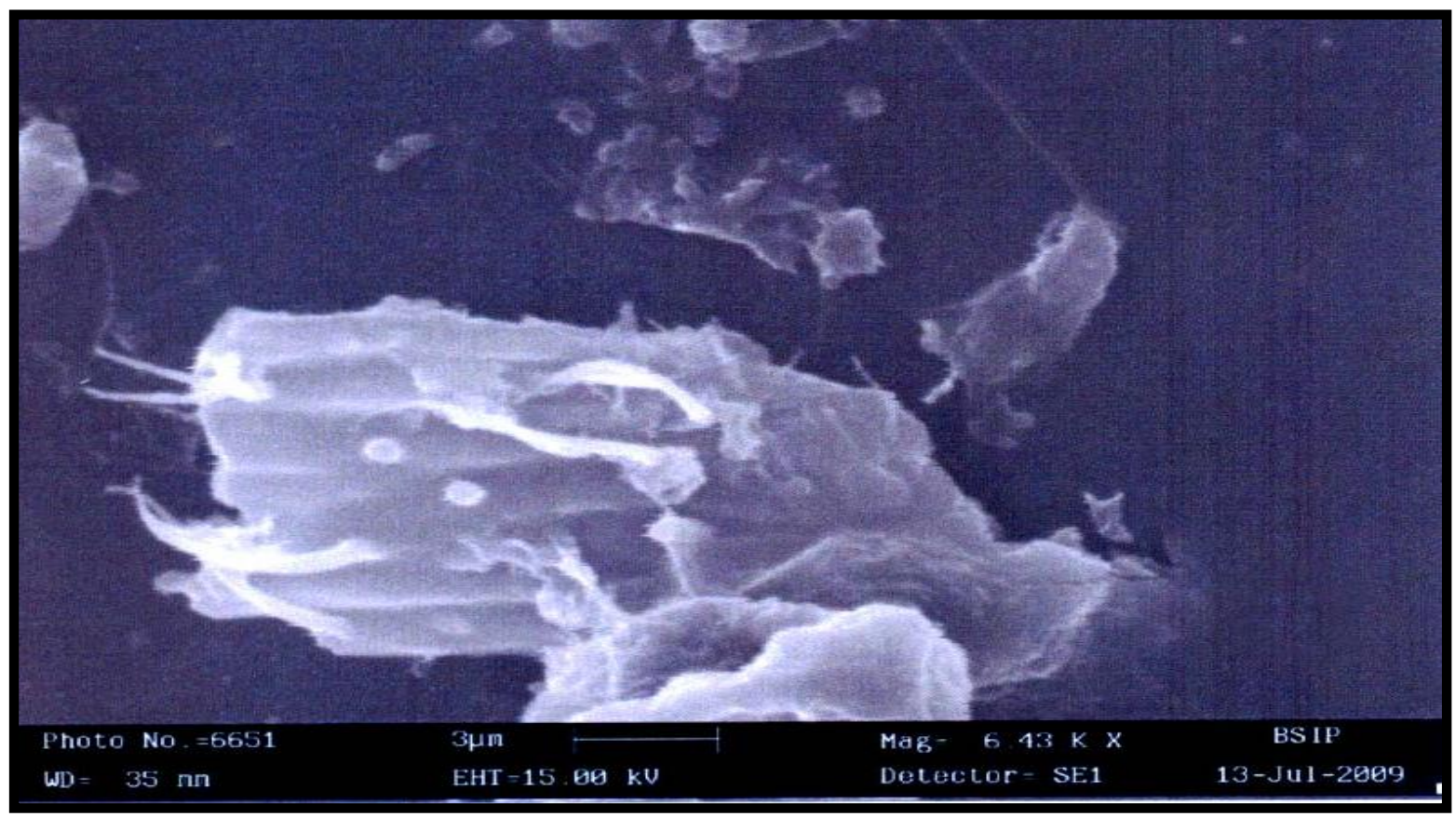

Figure 4 Shows SEM of Acanthamoeba polyphaga

able to survive freely in nature or endozoically inside mammalian hosts.It is because of this reason, samples were also taken from suspected patients of amoebic infection, who were using contaminated water for bathing, drinking, swimming and cleaning of their contact lenses. These amoebae also infect the eyes by using contaminated lenses or splashing unclean water. Infection has been known to be associated with wearing contact lenses during swimming (Stehr-Green et.al., 1989).

Acanthamoeba polyphaga (strain $\mathrm{E}_{1}, \mathrm{E}_{2}$ and $\mathrm{E}_{3}$ ) isolated from tears of eye. Marciano-Cabral et al., (2000) and Kilvington et al.,(1990) demonstrated the isolation of amoebae from a eye patient's cornea, contact lenses container, saline rinsing solution and kitchen cold tap water were identical, thus implicating domestic tap water as the source of Acanthamoeba sp. in Keratitis. Acanthamoeba have also isolated from CSF, human brain and nasal passage of healthy humans from different countries of world (Sadaka et.al., 1994; Shenoy et.al., 2002; Pisani et.al., 2003; Ahmad et.al., 2007; Kaushal et.al., 2008, Rai et.al., 2008 and Ahmad AlHerrawy et.al., 2014).)Amphizoic amoebae have also been reported from tears, corneal scrapings of eye patients (Sharma et.al. 2000; Schroeder et.al. 2001; Parija et.al. 2001; De Jonckheere, 2003, Kirwood, 2007, Safar, 2010 and Mafi M. et. al. 2014). Kilvington et.al.,(2004) demonstrated that isolates from a patients cornea contact lens container, saline rinsing solution, kitchen cold tap water were identified, thus the study implicated, for the first time, domestic tap water as the sources of Acanthamoeba species in keratitis. Chynn et.al (1997) also published an incidence of contaminated tap water exposure in Acanthamoeba keratitis patients. Khan and Paget (2002) have also reported the isolation of $A$. polyphaga from tap water in Leicester, U.K. Kamelet.al., (2004) first time reported a non contact lens related Acanthamoeba keratitis from Malaysia, in a 28 years old Indonesian male construction worker who had a trauma of the right eye during work. His eye was struck by sand and dust particles after which he quickly washed with water from a open tank at the constructions site.Tap water may be the origin of the infection via contact lenses and poor contact lens hygiene (Kilvington et. al., 2004).

The result of present study showed that clinical recognition of the signs and risk of factors for 
Acanthamoeba keratitis among non-contact lens wearer, as early diagnosis and prompt treatment are associated with improved clinical outcome.

Effective drug therapy was attributable to these free-living amoebae to cause corneal infection was improved and new drugs are needed, particularly when a resistance to classical therapeutics is encountered.

\section{Acknowledgment}

Authors are thankful to the Head, Department of Zoology, University of Lucknow, Lucknow for providing necessary facilities and encouragement.

\section{References}

Ahmad, K., Dil, A.S., Ahmad, I., Hussain, S. and Lakhnana, N.A. 2007. Primary Amoebic Meningoencephalitis: Report of a case. International Journal of Pathology. 5 (1): 34-36

Ahmad Al-Herrawy, Mahmoud Bahgat, Abd-Elhafez Mohammed,Ameen Ashour and WafaaHikal 2014. Acanthamoeba species in swimming pools of Cairo, Egypt. Iranian J. ParasitolVol. 9, No. 2, pp. 194-201.

Anisah, N., Yusof, S., Wan Norliana, A., Noraina, A.R. and Norhayati, M. 2004. Acanthamoebasp. Isolated from salt water in the west coast of peninsular Malaysia. Tropical Biomedicine. 21(1): 109-111.

Carter, R.F. 1968. Primary amoebic meningoencephalitis: clinical, pathological and epidemiological features of six fatal cases. Journal of Pathology and Bacteriology. 96:1-5.

Chynn, E.W., Talamo, J.H. and Seligman, M.S. 1997.Acanthamoeba keratitis: is water exposure a true risk factor? CLAO Journal 23(1): 55-56.

Culbertson, C.G., Ensminger, P.W. and Overton, W.M. 1972. Amoebic cellulocutaneous invasion by Naegleriaaerobia with generalized visceral lesions after subcutaneous inoculation: an experimental study in guinea pig. Am.J.Clin.Patj. 57:375-386.

De Jonckheere, J.F. 2003. Epidemiological typing of Acanthamoeba keratitis isolated from keratitis cases in Belgium. Bull. Soc. Belg. Ophthalmol.287: 27-33.

Elmeya H. Safar 2010. Involvement of the eye with Acanthamoeba.JASMR, 5(2): 191-198.

Hammersmith K.M. 2006. Diagnosis and management of Acanthamoeba keratitis. CurrOpinOphthalmol. 17:327331.

Kamel, A.G.M., Saleba, Anisah Nordin, Yusof Suboh, Norhayati Moktar and Norazah 2004. Isolation of
Acanthamoeba spp. Daripadapersekitaranakuatik. Prosiding Simposium Sains Kesihatan Kebangsaan Ke 5, Marriot Putrajaya, 25-26 Mei, 497-501.

Kaushal, V., Chhina, D.K., Ram, S., Singh, G., Kaushal, R.K. and Kumar, R. 2008. Primary Amoebic Meningoencephalitis due to Naegleriafowleri. JAPI,vol. 56.

Khan, N.A. and Paget, T.A. 2002. Molecular tools for speciation and Epidemiological studies of Acanthamoeba. Current Micro.44: 444-449.

Khan, N.A (2003). Pathogenesis of Acanthamoeba infections. Micob. Pathog. 34, 277-285.

Kilvington, S., Gray, T., Dart, J., Morlwt,N., Beeching, J.R., Frazer, D.G. and Matheson, M. 2004. Acanthamoeba keratitis The role of domestic tap water contamination in the United Kingdom. Invest. Opthamol. Vis. Sci. 45:165169.

Kilvington, S., Larkin, D.F.P., White, D.G. and Beeching, J.R. 1990. Laboratory investigation of Acanthamoeba keratitis. J. Clin. Microbiol.28: 2722-2725.

Kirwood, B.J. 2007. Acanthamoeba keratitis; an overview. The Journal of the American Society of Ophthalmic Registered Nurses, Ind. Vol. XXXII, No.3,16-20.

Levine, N.D.,Corless, J.O.,Cox, F.E.G., Deroux,G., Grain,J., Honigberg,B.M., Leedale,G.F., Loeblick, A.R., Lom,j., Lynn, D., Merinfeld, E.G.,Page,F.C.,Polijansky, G.,Sprague,V.,Vavra,J. Andwallance, F.C.1980. A newly revised classification of the protozoa. J.Protozool.27:3758.

Mafi M., Niyyati M., and Haghighi A. 2014. Evaluation of Acanthamoeba species from Tehran surface water sources using Real Time PCR Method. International Conference on Food Biological and Medical Sciences (FBMS-2014), Bangkok (Thailand): 69-71.

Maraciano-cabral, F.R., Puffenbarger, R. And Carbal, G.A. 2000. The increasing importance of Acanthamoebainfection.J. Eukaryot. Microbiol.47: 29-36.

Parija, S.C., Shiv Prakash, M.R., Rao, V.A. and Vellaniparambil, R.J. 2001.Acanthamoeba keratitis in Pondichery.J. Comun. Dis. 33(2): 126-129.

Pisani, F., Costa, C., Oteri, G. And Loli, A. 2003. Identification of amoebae in the CSF in a patient with memningoencephalitis.Journal of Neurosurgeryand psychiatry, 74: 1445-1446.

Rai R., Singh, D.K., Srivastava, A.K. and Bhargava, A. 2008. A case report of primary amoebic meningoencephalitis.Indian Pediatrics. 1004, vol. 45 
Sadaka, H.A., El-nassery, S.F., Abou, Samra, L.M. and Awadalla, H.N. 1994. Isolation and identification of freeliving amoebae from some water sources in Alexandria. Journal of the Egyptian Society of Parasitology, 24: 247 257.

Schroeder, J.M., Booton, C.G., Ingrid, J.H.I., David, S.V., Markus, B.M., Fuerst, A.P. and Byers, J.T. 2001. Use of subgenic $18 \mathrm{~S}$ ribosomal DNA PCR and sequencing for genus and genotype identification of Acanthamoeba from humans with keratitis and from sewage sludge. J. Clin. Microbiol. 39(5): 1903-1911.

Siddiqui, R.and Khan, N.A 2012.Biology and pathogenesis of Acanthampeba.Parasites and Vectors.5:6.doi:10.11861, 1756-3305-5-6.

Sharma, A.K., Sharma, U.D. and Singh, S. 2000. First case of Acanthamoeba keratitis in a contact lens wearer from India.Biol. Memoirs. 26(2): 44-47.

Shenoy, S., Godwin.W, Prashanth, H.V., Vidyalakshmi, K., Dhanashree, B. And Bharath, R. 2002. Primary Meningoencephalitis by Naegleriafowleri; first reported case from Mangalore, South India. J.Clin.Microbiol.40 (1):309-310
Shoff M, Rogerson A, Schatz S, Seal D. 2007 Variable responses of Acanthamoeba strains to three multipurpose lens cleaning solutions.Optom Vis Sci.84:202-207.

Shoff ME, Rogerson A, Kessler K, Schatz S, Seal DV. 2008 Prevalence of Acanthamoeba and other naked amoebae in South Florida domestic water.J Water Health. 6:99-104.

Singh, B.N. 1985. Free living soil amoebae as human pathogen. Proc. Indian Natn. Sci. Acad. B 51(3):297-312.

Singh, B.N. and Hanumaiah, V. 1979. Studies on pathogenic and nonpathogenic amoebae and the bearing of nuclear division and locomotive form and behaviour on the classification of order Amoebida. Monograph No.1 of the Association of Microbiologist of India. Indian $\boldsymbol{J}$. Microbiol.1-80.

Stehr-Green, J.K., Bailey, T.M. and Visveavara, G.S. 1989.The epidemiology of Acanthamoebakeratitis in the Unites States.Am. J. Ophthalmol. 107:331-336.

Visvesvara, G.S 2010. Free-living Amoebae as opportunistic Agents of Human Disease. Journal of Neuroparasitology.Vol. (1), Article IDN 100802, 1-13. 